\title{
Téoros
}

Revue de recherche en tourisme

\section{Les retombées de l'ouverture du pont de la Confédération sur le tourisme à l'île-du-Prince-Édouard}

\section{Marc LeBlanc}

Volume 17, numéro 3, automne 1998

URI : https://id.erudit.org/iderudit/1072182ar

DOI : https://doi.org/10.7202/1072182ar

Aller au sommaire du numéro

Éditeur(s)

Université du Québec à Montréal

ISSN

0712-8657 (imprimé)

1923-2705 (numérique)

Découvrir la revue

Citer cet article

LeBlanc, M. (1998). Les retombées de l'ouverture du pont de la Confédération sur le tourisme à l'île-du-Prince-Édouard. Téoros, 17(3), 58-64.

https://doi.org/10.7202/1072182ar d'utilisation que vous pouvez consulter en ligne. 


\section{H O R S T H E M E}

\section{LES RETOMBÉES DE L'OUVERTURE DU PONT de la ConfÉdération SUR LE TOURIŚme À
L'Île-DU-Prince-ÉdouARd}

\section{Marc LeBlanc}

Le pont de la Confedération a eu des retombées importantes sur l'industrie touristique de l'Tlle-du-Prince-Édouard. Le nombre de visiteurs a augmenté de $60 \%$ en une seule année et les craintes des intervenants touristiques ont vite été oubliées après ce départ fulgurant.

L'un des plus importants investissements financiers du siècle dans les provinces maritimes a eu des conséquences directes sur l'industrie du tourisme de l' l̂le-du-PrinceÉdouard. Il s'agit de l'ouverture, en mai 1997, du pont de la Confédération reliant cette province au continent, soit au Nouveau-Brunswick. L'ouverture du pont à la circulation est à l'origine d'une vigoureuse relance du tourisme de la province insulaire qui a accueilli, en 1997, 1189000 visiteurs ${ }^{1}$, ce qui représente une augmentation de $60 \%$ sur les entrées de $1996^{2}$.

Puisque l'évolution des moyens de transport influence, et même détermine, les flux touristiques ${ }^{3}$, il nous a semblé pertinent de présenter et d'évaluer les retombées touristiques à court terme de cette infrastructure. Notre évaluation portera essentiellement sur les conséquences que le trafic du pont a sur les différentes régions touristiques de l'île, tant en fonction du nombre de visiteurs que de diverses données et indicateurs significatifs.

L'une des difficultés rencontrées dans l'évaluation des retombées tenait à l'absence de données qui auraient permis de comparer l'influence du pont de la Confédération avec d'autres installations du mềme type, ailleurs au Canada ou dans le monde $e^{4}$.
Commençons par la petite histoire d'une grande aventure pour les insulaires.

\section{UNE HISTOIRE QUI NE DATE PAS D'HIER}

L'entrée de l'l̂le-du-Prince-Édouard dans la confédération canadienne, en 1873, fut en partie décidée à la suite de la garantie offerte par Ottawa d'assurer un lien entre cette nouvelle province et le continent. Dès 1877 , un bateau à vapeur assurait le transport de passagers entre l'île-du-PrinceÉdouard et le Nouveau-Brunswick. Quelques années plus tard, en 1885 , un sétnateur proposa le percement d'un tunnel sous le détroit de Northumberland. Si cette proposition fut refusée, elle eut du moins le mérite d'inciter le gouvernement fédéral à améliorer le transport en affectant à la liaison maritime un nouveau bâtiment à vapeur puissant $t^{5}$.

Vers la fin du XIX' siècle, en 1890 plus précisément, le gouvernement canadien entreprenait ses premières études de faisabilité du percement d'un tunnel. Le premier brise-glace opérant toute l'année fut mis en service en 1917. Il s'agissait du premier traversier pouvant transporter véhicules et passagers. En 1956, on éva- lua les possibilités de construction d'une chaussée joignant les deux rives du détroit. Une autre étape fut franchie en 1965 avec de multiples études approfondies sur les possibilités respectives de réalisation d'un pont, d'un tunnel ou d'une chaussée. Une fois de plus, les autorités décidèrent d'améliorer le service de traversiers.

Après d'autres études réalisées vers le milieu des années 1980 et à la suite d'un référendum tenu en 1988, qui recueillit $60 \%$ de votes des insulaires en faveur de la construction d'un pont, le projet prenait enfin corps. Finalement, des négociations entre la province et le gouvernement fédéral aboutissaient à la signature d'une entente entre les deux parties et à la création, le 7 octobre 1993, du consortium Strait Crossing Development Inc.

La construction du pont, commencée le 11 juillet 1994, se termina par la cérémonie d'ouverture officielle le 30 mai 1997. Le pont, d'une longueur de $12,9 \mathrm{~km}$ (dans le détroit de Northumberland, là où le climat hivernal rigoureux n'est qu'un des défis techniques qu'il a fallu relever) ${ }^{6}$ représente des investissements de l'ordre de 840 millions de dollars, entièrement financés par l'entreprise privée. Par ailleurs, le gouvernement canadien versera au concessionnaire une indemnité annuelle de 42 millions de dollars pendant 35 ans, non indexée sur le taux d'inflation, assurant des économies sur le service antérieur de traversiers dont le cout aurait suivi l'inflation (économies évaluées à 263,6 millions de dollars pour la durée du contrat ${ }^{7}$. En 2032, le gouvernement canadien deviendra l'unique propriétaire du pont de la Confédération dont la vie utile, selon les ingénieurs, serait de cent ans. 


\section{DES PRÉVISIONS À LA RÉALITÉ}

Des auteurs ont déjà évalué les retombées sur le tourisme de l'aménagement d'une autoroute dans des régions éloignées. Nous retiendrons deux constatations apparemment pertinentes à la situation de l'Île-duPrince-Édouard. D'une part, de telles infrastructures favorisent particulièrement les courts séjours de week-end ${ }^{8}$ et, d'autre part, les conséquences de l'ouverture d'une telle voie d'accès sont toujours plus perceptibles en milieu rural que dans une agglomération urbaine ${ }^{y}$. Ajoutons que le pont de la Confédération a été construit juste à proximité des anciennes gares maritimes du traversier. En effet, la nouvelle voie d'accès à partir du NouveallBrunswick est située à $5 \mathrm{~km}$ de l'ancien embarcadère, alors que du côtté de l' îledu-Prince-Édouard, c'est essentiellement le même emplacement, à quelques centaines de mètres de distance. Les visiteurs n'ont done pas à modifier leurs habitudes pour emprunter ce nouveau trajet, ce qui constitue un elément important. En effet, dans l'industrie touristique, on reconnaît généralement qu'il est avantageux que les. visiteurs conservent les habitudes acquises de trajets ${ }^{10}$.

Les études menées au cours des années 1980 avaient évalué plusieurs projets de lien fixe. Au moins deux ont plus spécialement porté sur les prévisions de fréquentation touristique subséquentes à l'ouverture d'un pont ${ }^{11}$. Les premières estimations tablaient sur une augmentation du nombre de visiteurs de l'ordre de 20 à $25 \%$ la première année de service, suivie d'une croissance annuelle de l'ordre de 2 a $3 \%$. Ces chiffres étaient basés sur la fréquentation des deux services de traversiers ${ }^{12}$ qui assuraient la liaison entre l'île et le continent, ainsi qu'en fonction des effets de la construction d'infrastructures majeures telles que les ponts Oland Island en Suède, le MacKinac Bridge au Michigan et le pont de la rivière Saint-Jean à Fredericton. Les auteurs relevaient également une augmentation de $42 \%$ dutant la première année et une croissance annuelle de $7 \%$ du trafic sur l'île du Cap-Breton après l'ouverture de la chaussée de Canso en Nouvelle-Écosse au début des années $1970^{13}$.

Le tableau 1 présente le nombre de visiteurs selon les premières prévisions et celles obtenues depuis l'ouverture du pont.

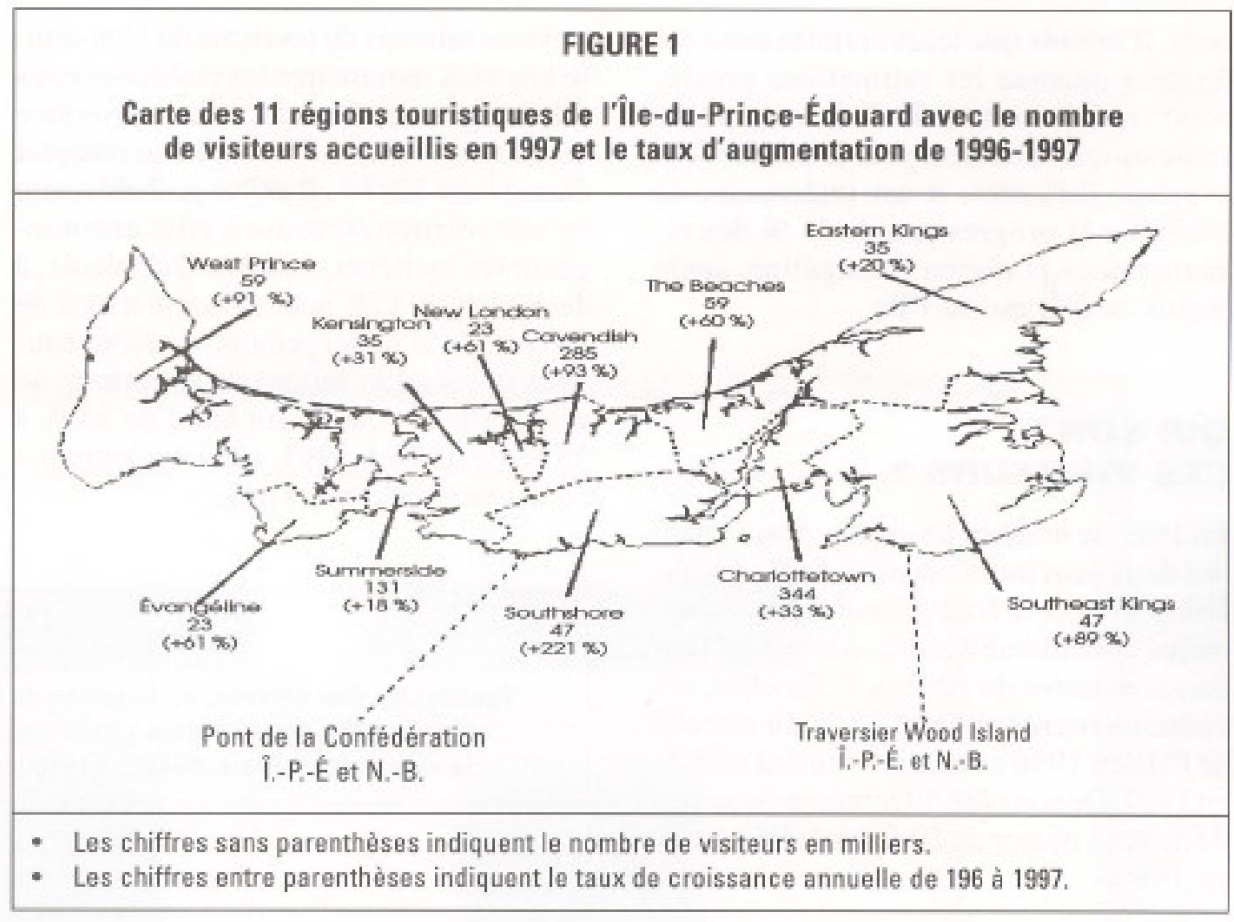

\begin{tabular}{|c|c|c|}
\hline \multicolumn{3}{|c|}{$\begin{array}{l}\text { Fréquentation touristique à lîle-du-Prince-Édouard, } \\
\text { prévisions et données réelles obtenues du } 14 \text { mai au } 31 \text { octobre } 1997\end{array}$} \\
\hline & $\begin{array}{l}\text { Dernière année de service } \\
\text { du traversier de Marine Atlantique }\end{array}$ & $\begin{array}{l}\text { Première année de service } \\
\text { du pont de la Confédération } \\
\text { (visiteurs) }\end{array}$ \\
\hline $\begin{array}{l}\text { Prévisions selon } \\
\text { Fiander-Good Associates } \\
\text { et Smith Green and Associates } \\
1989\end{array}$ & $\begin{array}{l}607000 \text { en } 1988 \\
725000 \text { fréquentation prévue } \\
\text { en } 1994\end{array}$ & $\begin{array}{l}870000 \text { en } 1995(+20 \%) \\
906000 \text { en } 1995(+25 \%)\end{array}$ \\
\hline $\begin{array}{l}\text { Données réelles fournies par } \\
\text { le gouvernement } \\
\text { de l'Île-du-Prince-Édouard }\end{array}$ & 741000 en $1996^{*}$ & 1189000 eก $1997(+60 \%)$ \\
\hline
\end{tabular}

On constate que le nombre de visiteurs est passé de 741000 à 1189000 de 1996 à 1997. Les responsables du tourisme de l'île attribuent cette augmentation au pont et au succès suscité par l'ampleur de l'ouvrage que certains qualifient de monument ${ }^{14}$.

Examinons, à l'aide de la carte géographique de l'île présentée â la figure 1, la répartition des visiteurs sur l'ensemble du territoire afin d'évaluer les effets réels de cette augmentation sur chacune des régions touristiques. Il n'est guère surprenant que la région de South Shore, où se situe la voie d'accès au pont, ait connu une très forte augmentation de ses visiteurs, de l'ordre de $221 \%$. Les trois régions traditionnellement les plus fréquentées par les visiteurs demeurent toujours en tête de liste quant au nombre absolu, soit : Charlottetown $(+33 \%)$, Cavendish $(+93 \%)$ et Summerside $(+18 \%)$.

Les responsables du service de traversiers entre Wood Island, dans la région Southeast Kings, et Caribou, en NouvelleÉcosse, avaient exprimé quelques inquiétudes quant aux conséquences de l'ouverture du pont sur la fréquentation de leurs navires. Ils craignaient de voir leur part de marché s'effriter si les visiteurs habitant la Nouvelle-Écosse préféraient utiliser le 
pont. Il semble que leurs craintes aient été fondées puisque les estimations préalables $^{15}$ qui fixaient à $50 \%$ le nombre de visiteurs qui opteraient pour le pont se sont avérées. En outre, il est intéressant de constater la progression de $61 \%$ des visiteurs dans la région Évangéline, seule région acadienne de l'île.

\section{QUI SONT CES VISITEURS ?}

En 1997, le nombre de visiteurs provenant des deux provinces voisines, le NouveauBrunswick et la Nouvelle-Écosse, a augmenté considérablement, comme en font foi les données du tableau 2 . En effet, ces visiteurs représentaient $25 \%$ du marché de l'île en 1996 comparativement a $35 \%$ en 1997. De son côté, l'Ontario a vu sa part de marché passer de $20 \%$ en 1996 a $25 \%$ en 1997.

Malgré une faible augmentation de $1 \%$ de leur part de marché, les Québécois ont été les plus nombreux (26\%) à visiter l'île, principalement pour voir le nouveau pont et $y$ \& rouler $\%$. La visite du pont influence également les visiteurs en provenance des Maritimes, $24 \%$ ayant déclaré que cette visite constituait le but principal de leur voyage.

Il est interessant de constater que les visiteurs en provenance des a autres lieux d'origine ne représentaient que $24 \%$ du marché en 1997 comparativement a $38 \%$ l'année précédente. Par ailleurs, le nombre de Japonais, friands de la maison d'Anne aux pignons verts du célèbre roman de Lucy Maud Montgomery, est limité en 1997 à 8500 , alors qu'ils étaient plus de 12000 en 1996.

Le tableau 3 permet de mieux connaître la durée moyenne des nuitées des visiteurs à l'île et la part du séjour total passée à l'île. L'une des préoccupations de l'industrie de l'hébergement de l'île était la baisse probable du nombre de nuitées dans la province subsćquente aux possibilités accrues de retour au Nouveau-Brunswick après une journée de visite. On constate que la durée moyenne du séjour à l'île a augmenté, passant de 4,1 à 4,2 nuitées. Malgré cette légère augmentation, un examen attentif permet de constater une réduction sensible des séjours des Québécois de 1996 (5,2 nuitées) à 1997 (2,8 nuitées). Incidemment, le rapport statistique annuel des services officiels du tourisme du NouveauBrunswick montre que les établissements d'hébergement du sud-est de la province affichaient un taux d'occupation complet durant l'été $1997^{16}$. Il existe probablement ici une relation de cause à effet encourageant les visiteurs, sauf les Québécois, à demeurer sur l'île pour éviter le risque de difficultés d'hébergement au NouveauBrunswick. La longueur moyenne du sćjour sur l'île, avec un bond de $31 \%$ a $35 \%$ de 1996 à 1997, tend également à confirmer cette hypothèse.
Le tableau 4 confirme que l'industrie hôtelière de la province insulaire a bénéficié de l'afflux d'un nombre impressionnant de visiteurs. Les taux d'occupation des points d'hébergement atteignent $92 \%$ en juillet et $97 \%$ en aout. Une telle performance est d'autant plus remarquable que le nombre d'unités de logement disponibles a augmenté de quelques centaines de 1996 à 1997 grâce à la construction de nouveaux hôtels et motels et à l'agrandissement d'établissements un peu partout dans la province.

\begin{tabular}{|c|c|c|c|}
\hline \multicolumn{4}{|c|}{$\begin{array}{l}\text { Ventilation des entrées, en fonction du lieu d'origine des visiteurs en } 1996 \text { et } 1997 \\
\text { et proportion des visiteurs ayant indiqué que le pont de la Confédération était } \\
\text { la raison principale de leur déplacement à l'Île-du-Prince-Édouard en } 1997\end{array}$} \\
\hline & $\begin{array}{r}\text { Proporti } \\
\text { des } \\
1996\end{array}$ & $\begin{array}{l}\text { en total } \\
\text { trées } \\
1997\end{array}$ & $\begin{array}{c}\text { Pont étant la raison principale } \\
\text { du déplacement }\end{array}$ \\
\hline $\begin{array}{l}\text { Visiteurs du Nouveau-Brunswick } \\
\text { et de la Nouvelle-Écosse }\end{array}$ & $25 \%$ & $35 \%$ & $24 \%$ \\
\hline Terre-Neuve & $2 \%$ & $2 \%$ & $15 \%$ \\
\hline Québec & $6 \%$ & $7 \%$ & $26 \%$ \\
\hline Ontario & $20 \%$ & $25 \%$ & $15 \%$ \\
\hline Nouvelle-Angleterre & $9 \%$ & $7 \%$ & $10 \%$ \\
\hline Autres lieux d'origine & $38 \%$ & $24 \%$ & N.D. \\
\hline
\end{tabular}

Source : Estimations fournies par l"agence gouvernementale Enterprise PEI, Research Division.

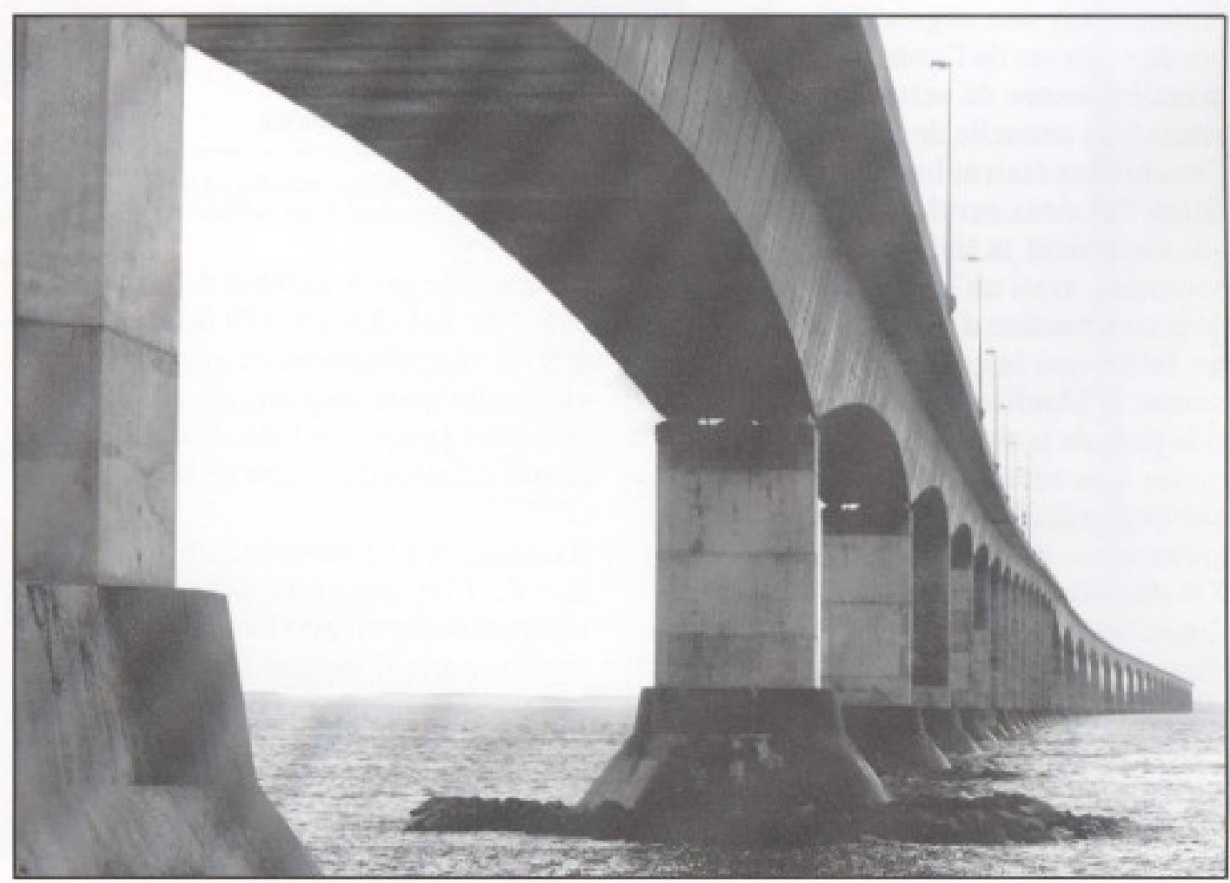


Les dépenses moyennes de séjour des groupes de visiteurs, présentées au tableau 5 , atteignaient 556 \&n 1997 , soit une hausse de $17 \%$ sur 1996. Les seuls groupes de visiteurs ayant réduit en moyenne leurs dépenses provenaient du Québec (de 560 \$ en 1996 à 426 \$ en 1997). Avec une part de marché de $2 \%$ (voir le tableau 2), les Terreneuviens ont néanmoins augmenté considérablement leurs dépenses moyennes pour atteindre la somme de 888 \$ par groupe en 1997, soit une augmentation de $37 \%$ par rapport à l'année précédente.

\section{L'ÉCONOMIE INSULAIRE FAVORISÉE PAR LE PONT}

Les dépenses totales des visiteurs à l'île, représentées au tableau 6, ont sensiblement augmenté depuis 1994, avec une progression marquée en 1997 ( $+54 \%$ ) pour un montant total record de 246 millions de dollars. Après un ralentissement en 1995 et 1996, le chiffre d'affaires des entreprises touristiques de l'île a done largement bénéficié de la présence du pont au cours de l'année 1997. C'est, sans aucun doute, le secteur de l'économie insulaire ayant connu la plus forte croissance en 1997.

Après avoir bénéficié largement des retombées attribuables aux travaux de construction du pont de 1993 à 1997, l'économie de l'île a aussitôt bénéficié de l'apport des revenus du tourisme correspondant à la forte hausse du nombre de visiteurs sur son territoire. En l'absence de données précises, on est en droit d'estimer, selon Rassi, que la construction du pont a produit les mêmes résultats qu'une manifestation importante, c'est-ă-dire que les effets d'une demande finale exogène portent non seulement sur la création d'emplois et l'apparition de nouveaux investissements, mais sur le niveau des revenus ${ }^{17}$. La nature du projet facilitant l'accès à l'île peut laisser croire que les retombées économiques sont plus importantes dans cette région touristique rurale qu'elles ne l'auraient été dans un milieu urbain ${ }^{18}$.

Il est intéressant de constater que le pont a aussi contribué à l'augmentation du nombre de visiteurs au Nouveau-Brunswick (selon les dirigeants du ministère responsable du Tourisme de cette province) : en hausse de $11 \%$ en 1997 pour atteindre 1343000 visiteurs comparativement à 1213000 l'année précédente. Lors de leur

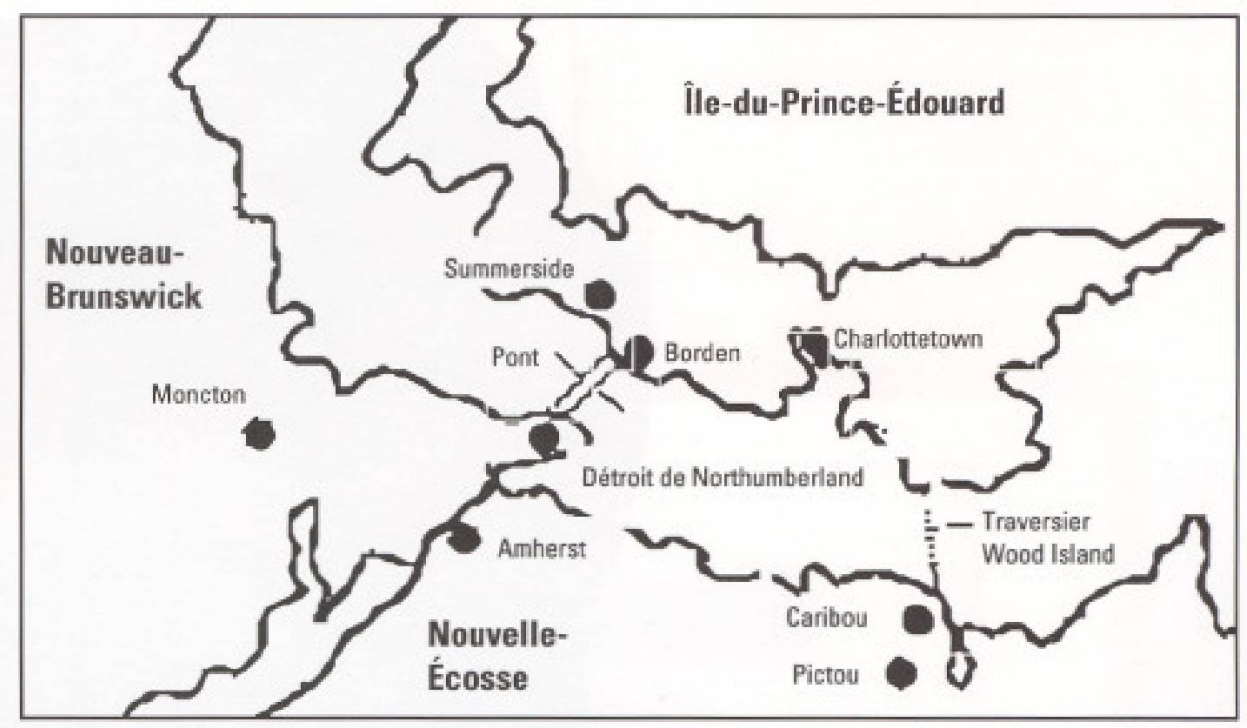

TABLEAU 3

\begin{tabular}{|lcc|}
\hline & \multicolumn{2}{c|}{ TABLEAU 3 } \\
& $\begin{array}{c}\text { Durée moyenne des nuitées et part du séjour total } \\
\text { passée âl l'île-du-Prince-Édouard de 1993 à 1997 }\end{array}$ \\
\hline & Nuitées moyennes à l'île & Part du séjour total passé à l'île \\
\hline 1993 & 4,8 & $42 \%$ \\
\hline 1994 & 4,6 & $38 \%$ \\
\hline 1995 & 4,8 & $37 \%$ \\
\hline 1996 & 4,1 & $31 \%$ \\
\hline 1997 & 4,2 & $35 \%$ \\
\hline
\end{tabular}

Source : Estimations foumies par l'agence gouvernementale Enterprise PEl, Research Division.

\section{TABLEAU 4}

Taux d'occupation des hôtels à lîle-du-Prince-Édouard en 1996 et 1997

\begin{tabular}{|lll|}
\hline & 1996 & 1997 \\
\hline Mai & $41 \%$ & $47 \%$ \\
\hline Juin & $71 \%$ & $74 \%$ \\
\hline Juillet & $90 \%$ & $92 \%$ \\
\hline Août & $93 \%$ & $97 \%$ \\
\hline Septembre & $60 \%$ & $63 \%$ \\
\hline Octobre & $61 \%$ & $60 \%$ \\
\hline
\end{tabular}

Source : Estimations foumies par l'agence gouvernementale Enterprise PEI, Research Division. 


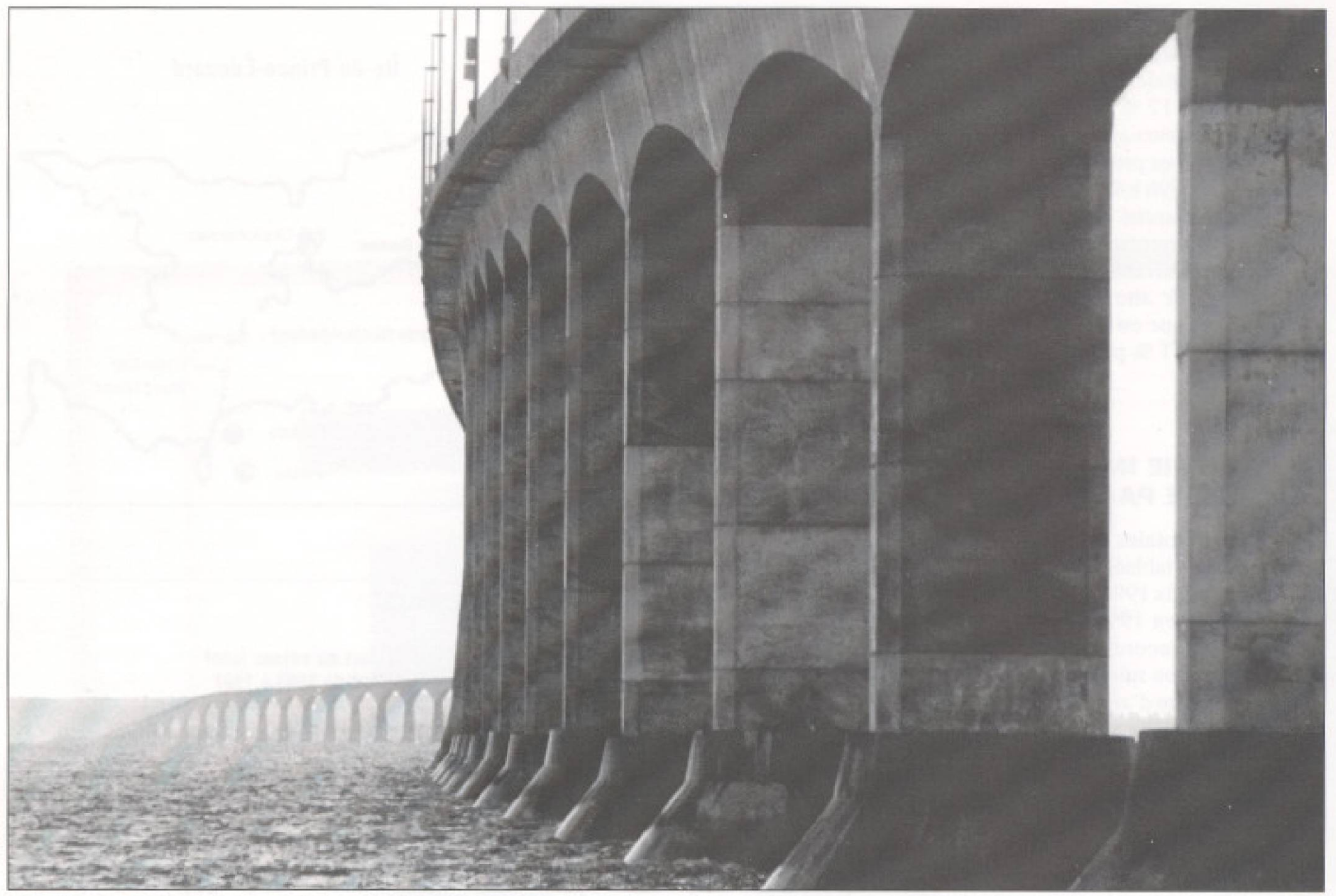

évaluation annuelle à l'automne 1997, les intervenants touristiques du sud-est du Nouveau-Brunswick, point d'accès au pont, ont exprimé leur vive satisfaction des résultats obtenus.

En revanche, la présence du pont a eu des retombées visibles sur l'industrie touristique des Illes-de-la-Madeleine où le nombre de visiteurs est tombé de 29000 en 1996 à 28000 en 1997. Toutefois, l'accroissement du nombre de visiteurs en provenance des provinces maritimes a compensé en partie la perte des visiteurs des autres régions du Québec ${ }^{19}$.

Par ailleurs, les habitants de l'ille n'auraient pas emprunté le pont pour quitter massivement leur province au cours de leurs vacances en 1997. C'est du moins ce que les responsables du tourisme insulaire laissent entendre. Dès 1989, Smith Grenne and Associates anticipaient cet êtat de fait et ajoutaient que la perte, si importante soitelle, du nombre de touristes intraprovin- ciaux serait largement compensée par le bond significatif (on parlait alors de $25 \%$ ) de visiteurs extérieurs à l'île. Avec une augmentation de $60 \%$, l'industrie touristique se réjouit et oublie, s'il y a lieu, les pertes des quelques insulaires partant vers d'autres cieux !

\section{QUE RÉSERVE L'AVENIR ?}

Les premières prévisions de $25 \%$ de taux d'augmentation des visiteurs étant d'ores et déjà largement dépassées, on est en droit de s'interroger sur l'évolution du taux de croissance espéré à compter de la deuxième année d'exploitation du pont, alors placé à 2 et $3 \%$ dans les prévisions. Les plus optimistes des entrepreneurs touristiques insulaires estiment que le nombre de visiteurs devrait marquer une nouvelle progression de 10 à $20 \%$ en 1998 , si l'on se fie à leurs déclarations au début de 1998 en réponse aux questions des médias régionaux concernant leurs prévisions d'ac- tivité durant la prochaine saison touristique.

Un projet de l'envergure du pont de la Confédération présente des caractéristiques si spécifiques que toute comparaison avec des réalisations similaires parait vaine. Déjà, le tunnel sous la Manche avait été en son temps soumis aux mêmes incertitudes et au contexte aléatoire de prévisions qui, selon Vickerman, procèdent essentiellement de trois facteurs : précision des données sur le trafic actuel et ses probabilités d'évolution à terme, fiabilité des hypothèses sur la structure du marché et caractéristiques de la future concurrence $^{20}$.

Les études diligentées annuellement par le ministère du Tourisme de l'île permettent de cerner les caractéristiques actuelles de l'activité dans le secteur du tourisme. Elles montrent un afflux de visiteurs en constante progression depuis plusieurs années, laissant entrevoir la poursuite de cette 


\section{TABLEAU 5}

\begin{tabular}{|lccc|}
\hline \multicolumn{4}{c}{ TABLEAU 5 } \\
& $\begin{array}{c}\text { Moyennes des dépenses par lieu d'origine des groupes } \\
\text { de visiteurs à l'île-du-Prince-Édouard en 1996 et 1997 }\end{array}$ \\
\hline & 1996 & 1997 & Variation \\
\hline Nouvelle-Écosse & $333 \$$ & $388 \$$ & $+17 \%$ \\
\hline Nouveau-Brunswick & $333 \$$ & $387 \$$ & $+17 \%$ \\
\hline Terre-Neuve & $648 \$$ & $888 \$$ & $+37 \%$ \\
\hline Québec & $560 \$$ & $426 \$$ & $-24 \%$ \\
\hline Ontario & $599 \$$ & $633 \$$ & $+6 \%$ \\
\hline Nouvelle-Angleterre & $533 \$$ & $590 \$$ & $+11 \%$ \\
\hline Total & $476 \$$ & $556 \$$ & $+17 \%$ \\
\hline
\end{tabular}

Source : Estimations fournies par I'egence gouvernementale Enterprise PEl, Research Division.

\section{TABLEAU 6}

Nombre de visiteurs et dépenses totales ainsi que leur croissance annuelle respective à I'T̂le-du-Prince-Édouard de 1993 à 1997

\begin{tabular}{|ccccc|}
\hline $\begin{array}{c}\text { Nombre de } \\
\text { visiteurs }\end{array}$ & $\begin{array}{c}\text { Croissance } \\
\text { annuelle }\end{array}$ & $\begin{array}{c}\text { Dépenses } \\
\text { totales }\end{array}$ & $\begin{array}{c}\text { Croissance } \\
\text { annuelle }\end{array}$ \\
\hline 1993 & 652000 & $+2,5 \%$ & $130000000 \$$ & \\
\hline 1994 & 668000 & $+2,3 \%$ & $146600000 \$$ & $+18,4 \%$ \\
\hline 1995 & 683000 & $+8,5 \%$ & $160100000 \$$ & $+9,2 \%$ \\
\hline 1996 & 741000 & $+60 \%$ & $245900000 \$$ & $+54 \%$ \\
\hline
\end{tabular}

Soerce : Estimations fournies par ragence gowernementale Enterprise PEI, Research Dwision.

tendance à court et moyen terme. En outre, la structure du marché, relativement bien connue, révèle un trafic de caractère plutôt régional principalement animé par les nombreux visiteurs en provenance du Nouveau-Brunswick et de la NouvelleÉcosse, provinces déjă concurrentes de l'île sur le marché du tourisme, enregistrant elles-mêmes de sensibles progressions des revenus dans ce domaine au cours des dernières années. La concurrence entre les trois provinces, de plus en plus vive, ne les empêche nullement de coordonner leurs efforts pour attirer chez elles un nombre toujours plus grand de touristes des provinces de l'Ouest canadien, des états de l'est des États-Unis et des pays d'Europe. Terre-Neuve a rejoint ces trois provinces pour coopérer aux programmes et à la promotion de la grande région atlantique.

De telles initiatives devraient normalement rapporter des dividendes à leurs secteurs respectifs d'économie. De ce point de vue, l'île jouit de la notoriété nationale particulière créce par la construction et l'ouverture du pont. Nous nous permettons ici un parallèle avec les auteurs d'évaluation des retombées touristiques à long terme, subséquentes a la tenue de manifestations exceptionnelles. A leur avis, de telles retom- bées peuvent se révéler plus importantes qu'initialement envisagé tout en contribuant à renforcer le prestige et l'image de la région concernée aux yeux des touristes potentiels ${ }^{21}$.

Dans leur article sur les facteurs d'interêt pour une région, $\mathrm{Hu}$ et Ritchie remarquaient que l'éloignement, les précédentes visites et le degré de connaissance de l'environnement favorisaient la bonne image d'une région $^{22}$. Compte tenu de la forte proportion des visiteurs en provenance du Nouveau-Brunswick et de la NouvelleÉcosse, dejà bien familiers avec les attractions et les sites de l'île, on peut estimer que ces visiteurs reviendront volontiers.

Enfin, les études préliminaires conduites avant la construction du pont ainsi que les travaux similaires exécutés durant la construction du tunnel sous la Manche aboutissaient à la conclusion suivante : toute nouvelle infrastructure, quelle qu'en soit l'importance, ne saurait se passer de projets d' * accompagnement $*$ tels que l'implantation d'espaces de loisirs et de commerces à proximité ${ }^{23}$. Les auteurs de ces études rejoignent ceux qui ont étudié les retombées à long terme du pont et qui ont conclu qu'il appartenait aux collectivités et aux entreprises locales d'assumer la conduite et l'orientation de cet $\alpha$ accompagnement $x^{24}$. Dans cette optique, il conviendrait d'analyser, à long terme, les conséquences de la qualité et de la disponibilité des hébergements hôteliers sur l'expérience des visiteurs.

Quelles seront, dans cinq ou dix ans, les répercussions du pont sur le tourisme dans l'île ? Des analyses périodiques de données devraient permettre d'en dégager les tendances. Sans préjuger de l'avenir, force est de constater le caractère extraordinaire des premières retombées et des brillantes perspectives ainsi ouvertes aux agents économiques du tourisme insulaire, aussi longtemps que ceux-ci sauront maintenir une coopération passant par une harmonisation des éventuels projets de développement.

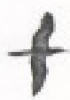

Manc LeBlanc est professeur agrégé à l'École d'éducation physique et de loisir de l'Université de Moncton. II se spécialise dans les domaines du tourisme et des communications pour le secteur récréatif. 


\section{NOTES}

1 Il s'agit ici du nombre d'entrés enregistrées du 14 mai au 31 octobre 1997, une estimation qui fait suite à un sondage de 3300 visiteurs. On notera que $85 \%$ du trafic touristique annuel se concentre à cette période.

2 Enterprise PEI, 1997 : 6.

3 Kaspar, 1985:12.

4 Delcan Stone and Webster, $1989: 5$; Smith Grenne and Associates, $1989: 3$.

5 J.L. Associates, 1997 ; 3.

6 Thurston, $1997: 54$.

7 Gardner Pinfold Consulting Economists Limited, $1993: 4$.

8 Kaspar, 1985:12.

9 Roehl et coll., $1993: 58$.

10 Gunn, $1988: 222$,

11 Fiander-Good Associates, 1987; Smith Green and Associates, 1989.

12 Marine Atlantique offrait, jusqu' à l'ouverture du pont le 31 mai 1997, une liaison entre Borden (Î.-P.-É.) et Cap Tourmentin (N.-B.). Une autre compagnie, la Northumberland Ferries Limited, assure toujours un service de transport de véhicules et de passagers entre Wood Islands (I..-P.-É.) et Caribou (N.-É.).

13 Smith Green and Associates, $1989: 16$.

14 Plante, $1997: 3$.

15 Smith Green and Associates, 1989: 26.

16 Planning and Research, $1997: 2$.

$171988: 5$.

18 Roehl et coll., $1993: 62$.

19 Association touristique des îles-de-la-Madeleine, $1997: 1$.

$201992: 242$.

21 Ritchie et coll., $1991 ; 3$.

$221993: 26$.

23 Spick, 1992 : 109 ; Breuillard-Pollet, 1992 : 341.

24 Stone and Webster, $1989: \mathrm{j}-4$.

\section{RÉFÉRENCES}

Association touristique des Îles-de-la-Madeleine (1997), Bilan de la saison 1997: Sommaire des résultats du sondage, Cap-aux-Meules.

Breuillard-Pollet, Michèle (1992), La dynamique du tunnel, dans Gérard Marcou, Roger Vinkerman et Yves Luchaire (dir.), Le tunnel sous la Manche entre états et marchés, Lille, Presses Universitaires de Lille, p. 329-378.

Delcan Stone \& Webster (1989), World Wide Literature Search, Halifax, Travaux publics Canada.

Enterprise PEI (1997), Economic Impact Tourism 1997, Charlottetown, Prince Edward Island, Research Division.

Fiander-Good Associates (1987), Economic Feasibility Assessment for the Northumberland Srait Crossing, Fredericton, Travaux publics Canada.

Gardner Pinfold Consulting Economists Limited (1993), A Benefit-Cost Analysis of the Northumberland Strait Crossing Project, Halifax, Travaux publics Canada.

Gunn, Clare (1988), Tourism Planning : Second Edition, New York, Taylor and Francis.

Hu, Yangzhou, et Brent Ritchie (1993), * Measuring Destination Attractiveness: A Contextual Approach », Journal of Travel Research, $\mathrm{n}^{\circ} 2$, automne, p. 25-34.

J.L. Associates (1997), Confederation Bridge Commemorative Edition: A Bridge in the Making 1993-1997, Port Elgin, N.-B.

Kaspar, Claude (1985), * L'interdépendance du tourisme et des transports en régions de montagne $*$, Revue de tourisme, $\mathrm{n}^{\circ} 2$, avril-juin, $\mathrm{p} .12$ 14.

Planning \& Research (1997), 1997 Tourism Industry Performance : Preliminary Year-End Forecast, Fredericton, Ministẻre du Développement économique et du Tourisme.

Plante, Gilles (1997), \& L'ouverture du Pont de la Confédération, l'événement de l'année $\%$ L'Acadie Nouvelle, 31 décembre, p. 3.

Rassi, Faouzi (1988), * Modèles de prévision et impact macro-économique d'une demande finale exogène ou d'un méga-événement *, Revue de tourisme, $\mathrm{n}^{\circ} 3$, juillet-septembre, p. 5-10.

Ritchie, Brent, et Brian Smith (1991) * The Impact of a Mega-Event on Host Region Awareness : A Longitudinal Study $\%$, Journal of Travel Research, $\mathrm{n}^{\circ} 1$, été, p. 3-10.

Roehl, Wesley, Julie Fesenmaier, et Daniel Fesenmaier (1993), * Hihgway Accessibility and Regional Tourist Expenditures $w_{\text {, Journal of }}$ Travel Research, $\mathrm{n}^{\circ} 3$, hiver, p. 58-63.
Smith Grenne and Associates (1989), Bridge Concept Assessment Supplement: Effect on Tourism and Associoted Development Strategies (Support Document for Question J), Halifax, Travaux publics Canada.

Smith Grenne and Associates (1989), Northumberland Strait Fixed Crossing : Inpacts on PEI Tourism, Halifax, Travaux publics Canada.

Spick, Jérôme (1992), Le unnel sous la Manche, Paris, Presses Universitaires de France.

Stone \& Webster (1989), Évaluation du projet de construction d'un pont : Supplement, Halifax, Travaux publics Canada.

Thurston, Harry (1997), \& Step Across the Strait *, Canadian Geographic, $\mathrm{n}^{\circ} 2$, mars-avril, p. $52-60$

Vickerman, Roger (1992), \& Le calcul économique *, dans Gérard Marcou, Roger Vinkerman et Yves Luchaire (dir.), Le tunnel sous la Manche entre états et manches, Lille, Presses Universitaires de Lille, p. 237-259 